\title{
Research, Data, and Administration Management in the Animal Health/One Health Era
}

James A. Guikema, Associate Vice President, Office of Research and Sponsored Programs, Kansas State University

$\mathrm{T}$ The theme of the current Merrill Conference - information systems as infrastructural priorities for university research both now and in the future - unites the research office and the information technology (IT) office at each university institution within our four-state region. There is an unprecedented demand on our IT infrastructure from nearly all sectors of our collective clientele. Students are sophisticated users of IT networks, and push the limits of what we can provide in communication, academic course content, and social networking. This is amplified when considering the needs of the distance-education arena. Our research-faculty investigators are developing larger databases that challenge our ability to archive, manage, manipulate, mine, and share essential information.

High on the list of research priorities are the 'omics' - genomics, proteomics, metabolomics, lipidomics, etc. with the i5K genome project [sequencing and annotating 5,000 insect genomes], described elsewhere in this volume, as an excellent example. Policy makers, and the managers of funding programs at both the state and federal levels, are sending our universities mixed messages regarding what data may (or must) be shared and what data must be secured.

Relative to IT demands, our universities are looking into the tunnel at the light - not knowing if that light is in fact the end of the tunnel, or if it is the headlight of the oncoming train. One thing is certain: a sense of a deadline approaching. This was perhaps best described by Dr. Samuel Johnson: "Nothing so concentrates the mind as the sight of the gallows," and the IT challenges are relevant across all disciplines on our campuses. Each of our insti- tutions has risen to the IT challenge in various areas and disciplines. This article seeks to place the IT / research infrastructure challenges within a 'comparative medicine' context, since these challenges touch upon research strengths of the region, of strengths within each institution, and of the growing regional opportunity represented by the relocation of a major federal comparative medicine laboratory [NBAF] to Manhattan, KS.

One Health / One Medicine: A 'comparative medicine' approach to cellular and molecular biology, as recognized within the United States, is an understanding that disease conditions, and the mechanisms which cause them, are similar within animal and human physiological systems. Thus, knowledge of one will enhance the understanding of the other. Creative research in either animal or human disease physiology will have a payoff in both sectors, implying common dis- 
ease mechanisms, common disease interventions, and common cures.

Although largely embraced by the academic community, implying a close linkage between colleges of medicine, veterinary medicine, and basic molecular biology, the comparative medicine viewpoint is often not recognized by the agencies which fund research. For example, the National Institutes of Health [NIH] demands a firm human health rationale for funding, and recent awards providing funding for the translation of research into the health enterprise do not consider animal health. Likewise, the National Science Foundation [NSF] will rarely fund a project with a human health rationale even at the basic molecular science level.

The comparative medicine approach is more widely embraced within agencies outside of the United States, with the addition of plant science to the human/animal/plant physiology and disease continuum. An example is the Australian National Biosecurity Flagship Program, which recognizes and funds infectious disease research on a broad level. Plant innate immunity protein homologs may play important roles in the physiology of Crohn's disease and the transfer mechanisms by which bacterial DNA is inserted into host cells were first documented in plant systems. Further, hunger is a looming political issue, with diseases such as wheat stem rust UG-99 and wheat blast taking center stage. In the past few months, there has been a $30 \%$ increase in the price of bread in Iran - a staple food in that country. Thus, the broader approach to comparative medicine recognizes food and water security as legitimate areas of research concern and funding.
Special Data Management Concerns in Research: Data management issues in research have resulted from the increasingly complex data sets routinely generated which are costly to archive, to mine, and to share. Our investigators recognize that, in collaborations which span institutions, making these data sets available to colleagues can create data-streaming challenges and require compatible assessment software on each side of the data-stream connection.

Federal funding agencies differ in their approaches to data sharing strategies. Two of the largest, NSF and NIH, have taken the strategy that, if data are to be useful for the good of society, they must be widely shared. NIH requires that all publications resulting from their funding be archived in PubMed. NSF has taken one step further, and requires the submission of a data management plan with every proposal for research funding. Kansas State University has responded to these requirements by providing a mechanism for submission to PubMed and by preparing for our investigators a data management plan which is suitable to NSF. The management plan varies depending upon 1] the size of the data sets, and 2] the frequency by which the research community will access them.

The Department of Defense, however, has taken a different approach and has proposed rules which will limit the sharing of DoD funded research. They are seeking limits on the rights of investigators to publish their results, and servers which house DoD-funded datasets may be required to be isolated with access controlled. If select agents are purchased with DoD funding, it is likely that expensive 
personnel surety measures must be in place for laboratory staff.

Research management also implies a concern for the welfare of the research environment. Welfare of the animal subjects is managed by the Institutional Animal Care and Use Committee established at each of our institutions. Welfare of the research staff member within the infectious disease research arena is a bit more complex, especially when the research involves pathogens considered by either the Centers for Disease Control (CDC) or the United States Department of Agriculture (USDA) to be a virulence threat to the investigator or a threat to the environment outside of the laboratory.

These two organizations have established physical specifications to meet laboratory safety goals, and these physical laboratory specifications are termed 'Biological Safety Levels (BSL)' defined as follows:

- BSL-1 - research involving pathogens which are not hazardous to investigators and which are not harmful to the environment if released.

- BSL-2 - research involving pathogens which may impact investigators in a non-serious way.

- BSL-3 - research with pathogens which may cause serious harm, but for which disease countermeasures exist.

- BSL-4 - research with pathogens for which no disease countermeasures exist.

- Facilities at the latter two levels require inspection by either CDC or USDA [or both], and obtaining per- mits for some work can take months or years.

Special Opportunities for the Four State Region: The four-state region represented by this conference is particularly strong in comparative medicine research activity. The greater Kansas City region, for example, is a hub of animal health economic activity, and has been dubbed the 'Animal Health Corridor'. Each of our research universities has strengths in the continuum from plant/animal/human disease physiology and has taken great strides to translate our research strengths into disease countermeasures useful in the wheat field, the cattle feedlot, or in the human hospital.

Our special strengths have also resulted in success in obtaining specialized facilities. The University of Missouri, for example, was successful in competing for a Regional Biocontainment Laboratory though an NIH competition. Ames, Iowa, is home to a USDA facility dedicated to animal health research, and the USDA Arthropod Borne Animal Disease Unit is located in Manhattan, Kansas. The State of Kansas has invested in the construction of the Biosecurity Research Institute, a BSL-3 laboratory facility associated with Kansas State University.

One tremendous opportunity looms on the horizon: the Department of Homeland Security plans to cease operations at the Plum Island Animal Disease Research Center located off the coast of Cape Cod, New York. In its place, DHS has planned the National Bio and Agro Defense Facility [NBAF] in Manhattan, Kansas, on land contiguous to Kansas State University. This facility will have both BSL-3 and BSL-4 laboratory capa- 
bilities, and is designed to protect the agricultural economic sector within the United States from foreign animal diseases.

Adding NBAF to the armamentarium of infectious disease research already within our four state region will provide us with facilities unmatched throughout the United States. 